\title{
EXTRACTION METHOD OF NON-RESTRICTIVE MODIFICATION IN JAPANESE AS A MARKED FACTOR OF PROSODY
}

\author{
Hisako Asano* Hisashi Ohara** Yoshifumi Ooyama*** \\ * NTT Information and Communication Systems Laboratories \\ ** NTT Technology Research Department \\ *** NTT Communication Science Laboratories \\ e-mail: hisako@nttnly.isl.ntt.jp
}

\begin{abstract}
This paper shows by a quantitative evaluation that nonrestrictive modification in Japanese is a marked factor of prosody, and describes how to extract non-restrictive modification. In Japanese, restrictive and non-restrictive modification have the same syntactic structure, so it is difficult to distinguish them. We propose rules to extract non-restrictive modification in news-text based on the classification of nonrestrictive modification and the combination patterns of the head noun and the modifier.
\end{abstract}

\section{INTRODUCTION}

Japanese text-to-speech conversion can be divided into text analysis processing, which gives Kanji (Japanese ideogram) readings and prosodic information (e.g. accentual phrase assignment, pause insertion etc.), and speech synthesis processing, which generates synthesized speech from the output of text analysis processing [2, 4]. Text analysis processing determines boundary information of each accentual phrase: that is, it determines whether there is a strong connection (SC), in which the fundamental frequency $\left(F_{0}\right)$ peak of the second phrase is lower than the first phrase, or a weak connection (WC), in which both phrases have approximately the same $F_{0}$ peak. Figure 1 shows $F_{0}$ contours of SC and WC.

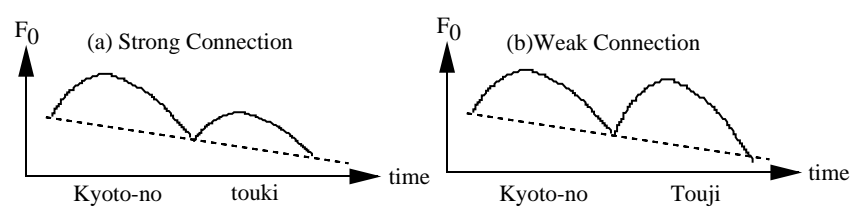

Figure 1: $F_{0}$ contours of $\mathrm{SC}$ and $\mathrm{WC}$

According to many studies $[3,4,6]$, there is an SC in the boundary when the first phrase modifies the second phrase, and a WC in the boundary when first phrase doesn't modify the second phrase. However, Kori [5] reports that if the modification is non-restrictive nominal modification, there is a WC in the boundary.

\author{
Kyoto-no [SC] touki (restrictive) \\ earthenware of Kyoto \\ Kyoto-no [WC] Touji (non-restrictive) \\ Touji (name of temple) in Kyoto
}

But there is no quantitative evaluation of the relation between non-restrictive modification and WC. Another problem is that non-restrictive modification cannot be clearly concluded because restrictive and non-restrictive modification have the same syntactic structure in Japanese.

This paper uses a quantitative evaluation to confirm that non-restrictive nominal modification is a critical factor of $\mathrm{WC}$, and determines the rules needed to extract nonrestrictive modification from news-text. The reasons for treating news-text are:

- In news-text, important words tend to be modified by extra information, and they apt to be non-restrictive.

- We are developing a Japanese text-to-speech system, AUDIOTEX [7], and the main target of AUDIOTEX is news-text.

Therefore, we aim to extract non-restrictive modification in news-text and assign WCs to synthesize easy-to-listen-to speech and improve the understanding of the content.

\section{2. $F_{0}$ ANALYSIS OF NEWS-TEXT}

\begin{tabular}{|l|l|r|}
\hline$(1)$ & $\begin{array}{l}\text { nominal modification in which } \\
\text { the modifier adjoins the head noun }\end{array}$ & 174 \\
\hline$(2)$ & non-restrictive modification in (1) & 30 \\
\hline$(3)$ & accentual phrases & 961 \\
\hline
\end{tabular}

Table 1: News-text data

We analyzed the $F_{0}$ of news-text of 2,493 words as uttered by a narrator. Table 1 shows the basic analysis of the news-text;

\footnotetext{
${ }^{1}$ We ignore the modification in which the noun phrase including the head noun becomes plural accentual phrases, because it is another factor of WC [1]. Therefore, we don't treat these noun phrases in extracting non-restrictive modification.
} 
the data indicating the relationship between non-restrictive modification and WC is shown in Table 2.

\begin{tabular}{|c|l|c|c|}
\hline$[1]$ & $\begin{array}{l}\text { WC in nominal modification } \\
\text { (WC in (1) of Table 1) }\end{array}$ & 63 & $36.2 \%$ \\
\hline$[2]$ & $\begin{array}{l}\text { WC in non-restrictive modification } \\
\text { (WC in (2) of Table 1) }\end{array}$ & 27 & $90.0 \%$ \\
\hline$[3]$ & $\begin{array}{l}\text { WC in non-restrictive modification / } \\
\text { WC in nominal modification }([2] /[1])\end{array}$ & - & $42.9 \%$ \\
\hline
\end{tabular}

Table 2: Non-restrictive modification and WC

Table 2 indicates that $90.0 \%$ of non-restrictive nominal modifications contain a WC, and these WCs account for the $42.9 \%$ of WCs in nominal modification. Thus we verify that non-restrictive modification is an important factor of WC in news-text.

\section{THE EXTRACTION METHOD}

Semantic analysis is generally needed to extract nonrestrictive modification because restrictive and nonrestrictive modification have same syntactic structure in Japanese. However, we use the classification of nonrestrictive modification and expression patterns in news-text gained from the word information of morphological analysis $[7,8]$ instead of deep semantic analysis.

First, we divide non-restrictive modification into 2 types; next, we show different extraction rules for each type.

\subsection{The Classification of Non- restrictive Modification}

We divide non-restrictive nominal modification into uniquedenotation modification and definitional modification based on uniqueness of the head noun.

In unique-denotation modification, the head noun is unique. For example:

$$
\text { Nihon-no Fuji-san (Mt. Fuji in Japan) }
$$

In definitional modification, the modifier denotes a property of the head noun, and the head noun need not be a unique object. For example:

jishin-no-kibo-wo-shimesu magnitude

(magnitude, which means the scale of earthquake)

\subsection{Unique-denotation Modification}

In unique-denotation modification, the head noun is almost always a proper noun. However, there are a few instances of restrictive modification which involves proper noun:

Christmas-no Fuji-san (Mt. Fuji on Christmas)
To extract unique-denotation modification with a proper noun, we must exclude such restrictive modification. We use Terashima's classification of modification [9], M-type-1 and M-type-2.

- M-type-1 distinguish the object which the head noun indicates from other objects.

e.g. tiisana byoin (a small hospital) [vs ookina byoin (a large hospital)]

- M-type-2 distinguish the state of the object which the head noun indicates from various states of the object. e.g. tiisai-toki-no Tom (Tom in childhood) [vs koukousei-jidai-no Tom (Tom in high school)]

These classifications are about any nominal modification, so we adapt them to non-restrictive modification, that is:

- NM-type-1 The modifier represents a property of the head noun, and the object which the head noun indicates is unique in terms of the property. This is the case of non-restrictive M-type-1.

- NM-type-2The modifier represents time or state. The head noun only exists at that time or the state. This is the case of non-restrictive M-type- 2 .

Based on NM-types, we analyzed semantic properties of proper nouns and modifiers. We classify proper nouns into 25 categories. Modifiers are classified into 3 patterns, which are further classified into 26 sub-patterns. The combinations of the proper noun categories and modifier sub-patterns determine the modification type.

First, we classified proper nouns into 25 semantic categories in terms of the proper noun's uniqueness (i.e. NM-type-1), and existent period (i.e. NM-type-2). Each category has a restriction priority or a non-restriction priority. Restriction priority is given to 2 categories which has low uniqueness, that is, language name and race or citizen name, and non-restriction priority is given to other 23 categories which have high uniqueness. A part of the classification - about organizations - is shown in Table 3 .

Next, we divided modifiers into the following 3 patterns based on NM-types. The 3 patterns are further divided into sub-patterns which consist of strings of word information, that is, the letters, parts of speech, semantic categories and so on. Some examples of the sub-patterns are indicated, where "numeral" stands for numerical expressions and ("numeral suffix") stands for optional numerical suffixes.

1. Definite restrictive pattern (4 sub-patterns) "numeral"-( "numeral suffix")-no (e.g. futatsu-no (two))

2. Time and state pattern (11 sub-patterns) "weather name"-no (e.g. ame-no (in the rain)) "numeral"- "time suffix"-( "suffix")-no (e.g. 80-nen-dai-no (in eighties)) 


\begin{tabular}{|l|l|l|}
\hline First category & Second category & Example \\
\hline \hline \multirow{2}{*}{ Hierarchical organization } & Top organization & NTT \\
\cline { 2 - 3 } & Subordinate organization & Tokyo-shiten (Tokyo branch) \\
\hline Plural area organization & - & Nihon-taishikan (Japanese embassy) \\
\hline Plural item organization & - & Nihon-shijo (Japanese market) \\
\hline Group organization & - & Nihon-kigyo (Japanese enterprise) \\
\hline Meeting & Single meeting & Tokyo-summit (Tokyo summit) \\
\cline { 2 - 3 } & Plural meeting & Beikoku-gikai (American Congress) \\
\hline
\end{tabular}

Table 3: A part of the classification(organization)

3. Default pattern (11 sub-patterns) "top organization"-no (e.g. NTT-no (NTT's))

Finally, the combination of the proper noun categories and modifier sub-patterns in each modifier pattern decides whether the modification is restrictive or non-restrictive.

1. Definite restrictive pattern In this case we treat the modification as restrictive, and don't extract the modification.

e.g. futari-no Nihon-jin (two Japanese)

2. Time and state pattern This case corresponds to Mtype-2, and we found 3 combinations for NM-type-2. For example:

The sub-pattern: any time and state pattern

The categories: single meeting, unique event, unique document or era name

e.g. 1991nen-no Wangan-senso (Gulf War in 1991)

The remaining combinations are treated as restrictive.

3. Default pattern This case corresponds to M-type-1, and we found 9 restrictive combinations which contain head nouns with the non-restrictive priority, and 1 nonrestrictive combination which contains restrictive priority head nouns. In these combinations, even if the proper noun category has non-restrictive priority, the modifications of restrictive combinations are restrictive, and even if it has restrictive priority, the modifications of non-restrictive combination are non-restrictive. The remaining combinations are determined to be restrictive or non-restrictive according to the priority of the head noun category contained in the combination. An instance of a restrictive combination is:

The sub-pattern: "top organization"-no

The category: subordinate organization

e.g. NTT-no Tokyo-shiten (NTT's Tokyo branch)

\subsection{Definitional Modification}

We analyzed the definitional modification in 10 days of newstext, and extracted the modifier expressions apt to be definitional modification. We determined that 3 types of nouns become the head noun in definitional modification, that is, compound words, loan words, and abbreviations.

The modifier expressions are the following 8 expressions.

$$
\begin{array}{ll}
\cdots \text {-wo-shimesu } & \text { (indicating } \cdots \text { ) } \\
\cdots \text {-wo-arawasu } & \text { (representing } \cdots \text { ) } \\
\cdots \text {-to-naru } & \text { (being } \cdots \text { ) } \\
\cdots \text {-de-aru } & \text { (being } \cdots \text { ) } \\
\cdots \text { to-iwareru } & (\text { said } \cdots \text { ) } \\
\cdots \text {-to-yobareru } & (\text { called } \cdots \text { ) } \\
\cdots \text {-nado-no } & (\cdots \text { and so on) } \\
\text { iwayuru } & \text { (what is called) }
\end{array}
$$

If the modification involves one of the three noun types and has a modifier expression, the modification is treated as definitional modification.

\subsection{The Flow of Extraction Process}

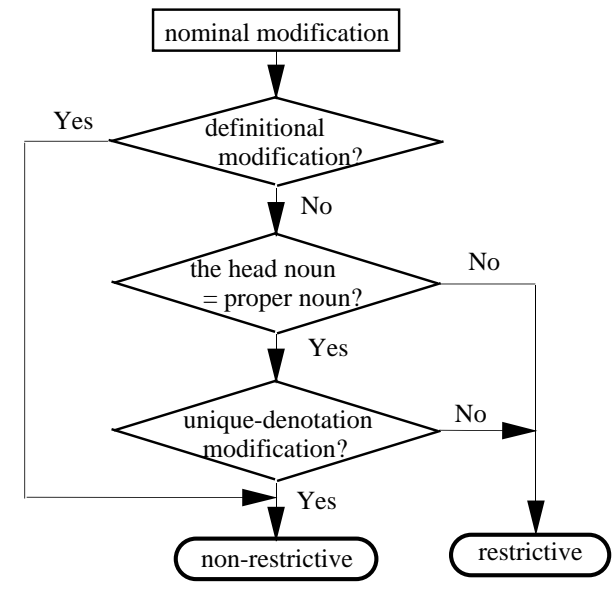

Figure 2: The flow of extraction process

Figure 2 shows the flow of extraction process. First, the process checks whether the modification is definitional modification, and next, if the head noun is the proper noun whether unique-denotation modification is involved. 


\begin{tabular}{|c|l|c|c|c|c|}
\hline \multirow{2}{*}{ No. } & Types of & \multicolumn{2}{|c|}{ Precision rate } & \multicolumn{2}{c|}{ Recall rate } \\
\cline { 3 - 6 } & modification & Expression & $(\%)$ & Expression & $(\%)$ \\
\hline \hline 1 & Unique-denotation & $\frac{A}{A+E}$ & 96.8 & $\frac{A}{A+B}$ & 95.8 \\
\hline 2 & Definitional & $\frac{C}{C}$ & 100 & $\frac{C}{C+D}$ & 63.0 \\
\hline \hline 3 & Total & $\frac{A+C}{A+C+E}$ & 97.6 & $\frac{A+C}{A+B+C+D}$ & 85.1 \\
\hline
\end{tabular}

Table 5: Precision rate and recall rate

\begin{tabular}{|c|c|c|c|c|}
\hline No. & \multicolumn{2}{|c|}{ Types of modification } & \multicolumn{2}{|c|}{$\begin{array}{c}\text { Extract as } \\
\text { non-restrictive? }\end{array}$} \\
\hline$\overline{\mathrm{A}}$ & \multirow{4}{*}{$\begin{array}{l}\text { Non-restrictive } \\
\text { modification }\end{array}$} & \multirow{2}{*}{$\begin{array}{l}\text { Unique- } \\
\text { denotation }\end{array}$} & Yes & $\overline{\overline{91}}$ \\
\hline $\mathrm{B}$ & & & No & 4 \\
\hline $\mathrm{C}$ & & \multirow[t]{2}{*}{ Definitional } & Yes & 29 \\
\hline $\mathrm{D}$ & & & No & 17 \\
\hline $\mathrm{E}$ & \multirow{2}{*}{\multicolumn{2}{|c|}{$\begin{array}{l}\text { Restrictive } \\
\text { modification }\end{array}$}} & Yes & $3^{2}$ \\
\hline $\mathrm{F}$ & & & No & 1771 \\
\hline
\end{tabular}

Table 4: The extraction result

\section{EVALUATION AND RESULT}

\subsection{Evaluation Data}

We evaluated the extraction rules using a news-text containing 34,722 words. The news-text contained 120 proper nouns of head nouns; 100 (83.3\%) of them were non-restrictive. The results are shown in Table 4 . We calculated the precision rate and recall rate as shown in Table 5. In Table 5, the capital letters in expression mean the item number of Table 4.

\subsection{Unique-denotation Modification}

Unique-denotation modification has high precision rate and recall rate. The reason of wrong extraction is an insufficient variety of restrictive combinations. For example, "Naritahatsu-no Nikkoki (a JAL plane leaving Narita)" is restrictive modification, but this modification doesn't match restrictive combinations in default pattern.

\subsection{Definitional Modification}

Definitional modification has perfect precision rate. However, the recall rate is a little low because the extraction rules don't use semantic analysis which is required to identify some instances of the definitional modification. For example, "tei-kougai-no methanol (methanol, which is lowpollution)" is non-restrictive modification, but we must know that "methanol" offers little pollution to extract this modification, because all modifiers consisting of noun phrases have a same last word " $n o$ ".

\footnotetext{
${ }^{2}$ All of them are extracted as unique-denotation modification.
}

\section{CONCLUSION}

We show non-restrictive modification is a factor of WC, and decide rules to extract non-restrictive modification using word information of morphological analysis. These rules can extract $85.1 \%$ of non-restrictive modification instances, and extract $97.6 \%$ right non-restrictive modification instance. Hereafter, we will evaluate how much the rules improve the synthesized speech quality by a listening experiment, and study other factors of WC.

\section{REFERENCES}

1. Hisako Abe and Hisashi Ohara. Assignment of accentual phrase information based on restrictive and nonrestrictive modification (in Japanese). In SIG-SLUD9401, 1994 .

2. Kazuo Hakoda, Tomohisa Hirokawa, Hajime Tsukada, Yuki Yoshida, and Hideyuki Mizuno. Japanese textto-speech software based on waveform concatenation method. In Proc. AVOIS'95, 1995.

3. Kazuo Hakoda and Hirokazu Sato. Prosodic rules in connected speech synthesis (in Japanese). Trans. IEICE, J63$\mathrm{D}(9), 1980$.

4. Hisashi Kawai, Keikichi Hirose, and Hiroya Fujisaki. Rules for generating prosodic features for text-to-speech synthesis for Japanese (in Japanese). Journal of ASJ, 50(6), 1994.

5. Shiro Kori. Autonomy of prosody (in Japanese). Gengo(Language), 21(9), 1992.

6. Haruo Kubozono. The Organization of Japanese Prosody. Kurosio Publishers, 1993.

7. Kouji Matsuoka, Eiji Takeishi, Hisako Asano, Ryomi Ichii, and Yoshifumi Ooyama. Natural language processing in a Japanese text-to-speech system for written-style texts. In Proc. IVTTA'96, 1996.

8. Yoshifumi Ooyama, Masahiro Miyazaki, and Satoru Ikehara. Natural language processing in a Japanese text-tospeech system. In ACM Fifteenth Annual Computer Science Conference, 1987.

9. Hideo Teramura. Syntax and Meaning in Japanese II (in Japanese). Kurosio Publishers, 1984. 